\title{
Diffusion and reptation quantum Monte Carlo study of the NaK
}

molecule

Marc E. Segovia, Oscar N. Ventura*

CCBG, Detema, Faculty of Chemistry, UdelaR, CC1162, 11400 Montevideo, Uruguay

*Corresponding author: Oscar N. Ventura

CCBG-Detema

Facultad de Química, Udelar

Isidoro de María 1610, 4th floor

11400 Montevideo, Uruguay

Tel: $\quad+59899501368$

E-mail Oscar.N.Ventura@gmail.com

Orcid https://orcid.org/0000-0001-5474-0061

Twitter @ONVentura 


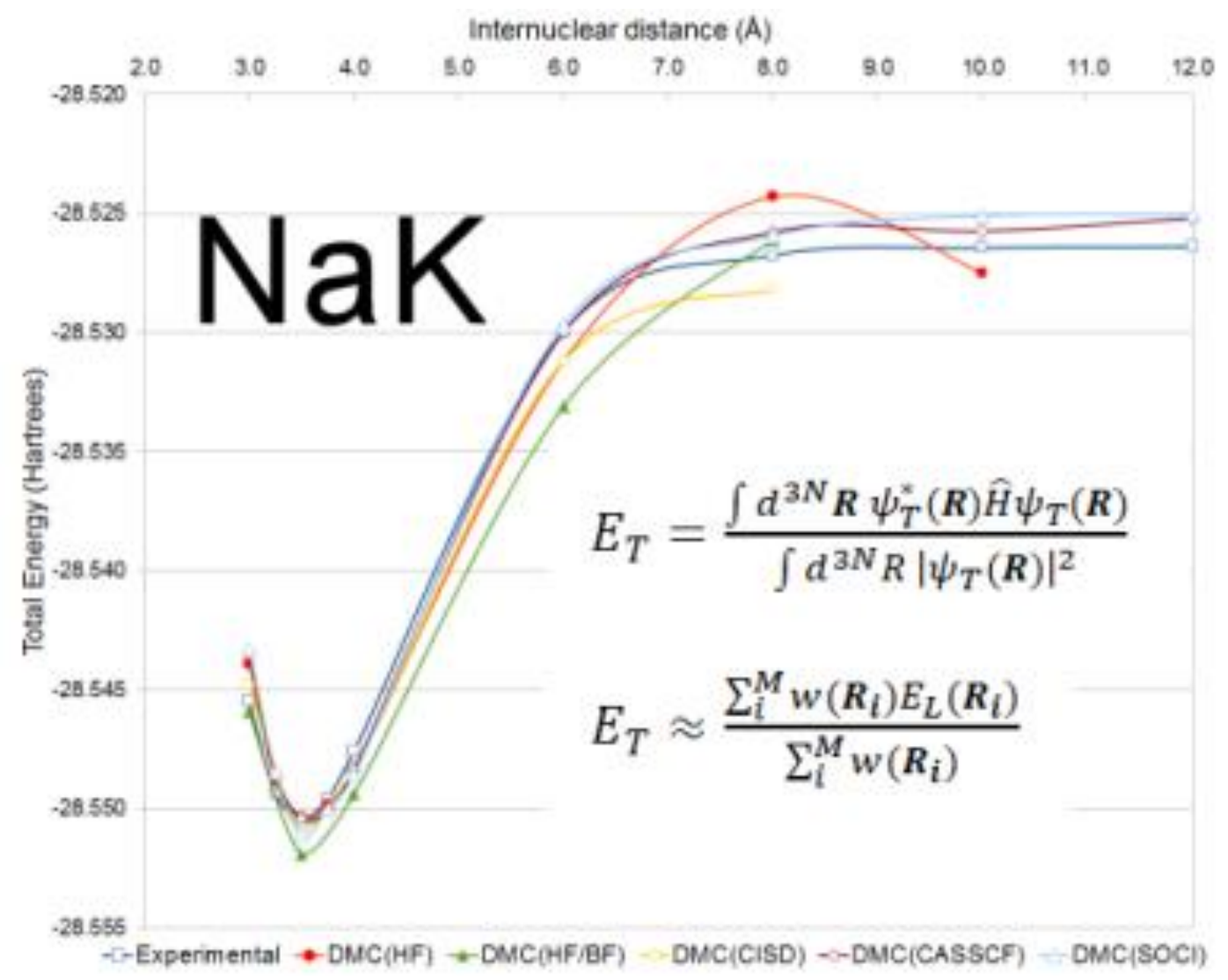




\section{Diffusion and reptation quantum Monte Carlo study of the NaK molecule}

Diffusion Monte Carlo (DMC) and Reptation Monte Carlo (RMC) methods, have been applied to study some properties of the NaK molecule. Hartree-Fock (HF), Density Functional (DFT) and single and double configuration interaction (SDCI) wavefunctions with a valence quadruple zeta atomic natural orbital (VQZ/ANO) basis set were used as trial wavefunctions. Values for the potential energy curve, dissociation energy and dipole moment were calculated for all methods and compared with experimental results and previous theoretical derivations. Quantum Monte Carlo (QMC) calculations were shown to be useful methods to recover correlation in $\mathrm{NaK}$, essential to obtain a reasonable description of the molecule. The equilibrium distance-interpolated from the potential energy curves-yield a value of $3.5 \AA$, in agreement with the experimental value. The dissociation energy, however, is not as good. In this case, a conventional $\operatorname{CCSD}(\mathrm{T})$ calculation with an extended aug-pc-4 basis set gives a much better agreement to experiment. On the contrary, the CCSD(T), other MO and DFT methods are not able to reproduce correctly the large dipole moment of this molecule. Even DMC methods with a simple HF trial wavefunction are able to give a better agreement to experiment. RMC methods are even better, and the value obtained with a B3LYP trial wavefunction is very close to the experimental one.

Keywords: quantum monte carlo, dipole, dissociation energy

\section{INTRODUCTION}

\section{Heading 1: use this style for level one headings}

One of the technologies that will shape our not so distant future-along virtual reality, artificial intelligence, androids and the like—will be quantum computing. Some years ago, Yelin et al. 1 suggested schemes for robust quantum computation using polar molecules. More recently, Kuztnetsova 2, in the same group, analyzed the experimental feasibility of polar molecule based phase gates, determining the requisites for a polar 
molecule qubit system. In their study they used the $\mathrm{CO}$ molecule, but other lines of research have pointed toward alkaline molecules..

New experimental techniques for the formation of polar molecules in their rovibrational ground states, namely stimulated rapid adiabatic passage (STIRAP 3) and Feshbach-optimized photo-association (FOPA 4), have allowed the study of ultracold $(\mathrm{v}=0)$ uncommon alkali diatomics, like LiCs 5 , KCs 6 or $\mathrm{KRb} 7$. There has been increased interest in the last ten years on these ultracold (formed below $1 \mathrm{mK}$ ) molecules, since their unusually large dipole moment allow them to be controlled by electric fields, promising new applications in quantum computing, ultracold chemistry, new phases of matter and precision measurements. Several theoretical studies have also appeared on the structure and properties of these molecules, a difficult task because the bond is a relatively weak van der Waals interaction, with large equilibrium bond distances and dipole moments and small dissociation energies8-19.

$\mathrm{NaK}$ is one of the heteronuclear alkali diatomic molecules that had been thoroughly investigated in past years. Ross 8 published a study of the ground $\mathrm{X} 1 \Sigma^{\wedge}+$ state using Fourier-transform infrared spectroscopy, while Wormsbecher 9 and Yamada 10 performed high resolution microwave spectroscopy experiments, obtaining values of 3.4990348(15) $\AA$ for the equilibrium distance (confirmed later by LIF experiments 11) and $2.73 \mathrm{D}$ for the dipole moment. The dipole moment of $\mathrm{NaK}$ had been previously determined experimentally as $\mu 0(39 \mathrm{~K} 23 \mathrm{Na})=2.76 \pm 0.1 \mathrm{D}$ by Dagdigian and Wharton 12.

It was later shown that the potential energy curve (PEC) derived by Ross from his experimental results was faulty. Russier-Antoine, Ross et al. reanalyzed the data and derived an improved PEC in 2000 13. The dissociation energy estimated in this work was $5273.78 \pm 0.24 \mathrm{~cm}-1(24.029 \pm 0.001$ mhartrees $)$. Gerdes et al. 14 performed recently 
a simultaneous analysis of the lowest $\mathrm{X} 1 \Sigma+$ and a3 $\Sigma+$ electronic states of the $\mathrm{NaK}$ molecule, confirming the equilibrium distance $\mathrm{Re}=3.4990 \AA$ and a dissociation energy of $5273.62 \pm 0.10 \mathrm{~cm}-1(24.0284 \pm 0.0004$ mhartrees $)$.

Some recent theoretical research has been performed also on this molecule. On the one side, Zemke et al. used the $\operatorname{CCSD}(\mathrm{T})$ method with the McLean-Chandler 6$311+\mathrm{G}(3 \mathrm{df})$ basis set to calculate the interaction of $\mathrm{Na} 2, \mathrm{~K} 2$ and NaK dimers 15 . Their calculations afforded values of $3.488 \AA$ for the equilibrium distance and $2.94 \mathrm{D}$ for the dipole moment of $\mathrm{NaK}$. Another important paper on this subject was published by Byrd et al 16. They used also the CCSD(T) method, but with the explicitily correlated F12b theory — which allows for greater precision - for the study of $\mathrm{K}$ and Rb clusters. Byrd et al. studied also the PECs of homonuclear alkali metal diatomics 17 using single excited configuration interaction (CIS) and time dependent density functional methods (TDDFT) using the B3PW91 and PBE0 potentials. Aymar and Dulieu 18 used a 7s5p5d2f $/[5 s 5 p 3 d 2 f]$ contracted basis set for $\mathrm{Na}$ and a $7 \mathrm{~s} 5 \mathrm{p} 7 \mathrm{~d} 2 \mathrm{f} /[6 \mathrm{~s} 4 \mathrm{p} 4 \mathrm{~d} 2 \mathrm{f}]$ contracted basis set for $\mathrm{K}$ in order to perform $\mathrm{CI}$ calculations of the dipole moment, including a pseudopotential to represent the atomic cores and a phenomenological potential to describe core polarization. They found a dipole moment of $2.76 \mathrm{D}$ at an optimum equilibrium distance of 3.440 Å. Finally, Fedorov et al. have recently determined accurate potential energy, dipole moment curves, and lifetimes of vibrational states of heteronuclear alkali dimers, including NaK 19 . They used all-electron quadruple- $\zeta$ basis sets with additional core functions for sodium, and small-core relativistic effective core potentials (ECP) with quadruple- $\zeta$ quality basis sets for potassium. An important conclusion of their work is that non-perturbative triples (i.e. CCSDT) are necessary for obtaining quantitative agreement between calculated and experimental results. 
In this work we aimed to describe the bond length, dipole moment and dissociation energy of the $\mathrm{NaK}$ molecule from two complementary points of view. On one side, we performed DFT calculations (PBE0) with and without an empirical dispersion correction, as well as $\operatorname{CCSD}(\mathrm{T})$ calculations to study the PECs of $\mathrm{NaK}$ and some of its properties at equilibrium, in order to stablish the link between former theoretical calculations and our own. On the other side, we used three versions of the quantum Monte Carlo (QMC) method, namely Diffusion Monte Carlo (DMC), Reptation Monte Carlo (RMC) and Variational Monte Carlo (VMC) to study the NaK molecule ground state. The QMC calculations used trial wavefunctions calculated at the monoconfigurational (Hartree-Fock) and multiconfigurational (MCSCF and CISD) levels, as well as several DFT methods (B3LYP, PBE and BHHLYP). PECs, equilibrium distances, dipole moments and dissociation energies were calculated and compared both with experimental values and results from other theoretical calculations. The results show that the VMC calculations combined with DMC give a result for the dissociation energy (23.0 and 25.2 mhartrees at the CISD and CASSCF based QMC levels) not very far from the experimental result (about $4 \%$ statistical error). RMC is necessary however to improve the agreement of the calculated dipole moment with the experimental value. Systematic improvements in QMC are observed when using multideterminant wavefunctions, or fixed node approximations (FNA) improvements such as backflow. Possible defficiencies due to the use of pseudopotentials and small basis sets are discussed. 


\section{COMPUTATIONAL METHODS}

\section{Conventional calculations}

Molecular orbital and DFT methods were used for calculating the potential energy curves (PEC) and to compare them with previous studies. Hartree-Fock (HF), CCSD(T), CASSCF and MRCI procedures were employed, using mainly the Kalsruhe Def2-QZVPPD basis set 20, both for Na and K. All electron (i.e. no pseudopotential) calculations were performed, but only valence electrons were correlated at the postHartree-Fock level of the PEC. All electrons were included however, in the CASSCF and MRCI calculation of the optimum equilibrium distance and properties at this geometry. DFT calculations of the PEC were performed using the PBE0 method 21 with and without the GD3 empirical dispersion correction by Grimme 22. Dissociation energies were obtained from the curves with respect to the asymptotic limit, both to avoid the need of BSSE corrections and the spin-orbit corrections due to the radical nature of the $\mathrm{Na}$ and $\mathrm{K}$ atoms. Geometry optimizations were performed to obtain the equilibrium distance and the dipole moment of the corresponding structure. All conventional calculations were performed using the G09 23 and Molpro 24 set of computer programs. Basis set dependency of the calculations was tested for the PBE0 and CCSD(T) methods employing the Def2-QZVP and Def2-TZVP Kalsruhe basis sets, as well as Feller quintuple-zeta pc-4 basis set 25, 26.

\section{Heading 2: use this style for level two headings}

QMC calculations were performed on the $\mathrm{NaK}$ starting from the experimental bond length. The QMC method is able to give an upper bound of the true ground state energy if the fixed-node approximation (FNA) is used (necessary to maintain the fermionic nature of the wave function). 27, 28, 29, 30, 31, 32 Thus, fixed-node diffusion MonteCarlo is the most common variant of QMC in use 33, 34. DMC calculations are started 
using a trial function, which can be a single Slater determinant or a multideterminantal wavefunction 35 . This function can be obtained either from molecular orbitals or from DFT Kohn-Sham orbitals. If the nodal surface of the trial wave function were exact, then the DMC method, if run for a large enough number of steps and extrapolated to zero timestep, would give the exact ground state energy. This is obviously not the case when using a single determinant MO wavefunction, although for some systems it may be a good approximation. All the QMC simulations in this work were done using an energy-consistent scalar-relativistic Hartree Fock pseudopotential (BFD) 36 with a VQZ/ANO basis set, using HF and a pair of DFT functionals (B3LYP, PBE) to build the trial wavefunction. The influence of a multideterminantal wavefunction was explored by CASSCF $(10,10)$ calculations, on top of which a second order configuration interaction (SOCI) — including all single and double excitations — was performed.

As a brief summary of the QMC method used in this paper, we will follow the description given by Kim et al 37. Given an N-electron system in a state described by a $3 \mathrm{~N}$-dimensional wave function $\Psi\left(\mathrm{r}_{-} 1, \ldots, \mathrm{r}_{-} \mathrm{N}\right)$ satisfiying the usual time-independent Schrödinger equation, $\mathrm{H} \Psi=\mathrm{E} \_0 \Psi$, with a hamiltonian including the kinetic and potential energy of interaction of the particles, QMC methods employ a trial function $\Psi_{-} \mathrm{T}$, which takes the general form

$$
\Psi_{T}(\boldsymbol{R})=\prod_{k} \psi_{k}(\{\alpha\}, \boldsymbol{R})
$$

where the $\psi_{-}$k are analytical functions depending on the optimizable parameters $\{\square\}$. The energy of the system can then be evaluated as

$$
E_{T}=\frac{\int d^{3 N} \boldsymbol{R} \psi_{T}^{*}(\boldsymbol{R}) \widehat{H} \psi_{T}(\boldsymbol{R})}{\int d^{3 N} R\left|\psi_{T}(\boldsymbol{R})\right|^{2}}
$$


This is a general equation, which in the case of QMC is estimated by stochasting sampling as

$$
E_{T} \approx \frac{\sum_{i}^{M} w\left(\boldsymbol{R}_{i}\right) E_{L}\left(\boldsymbol{R}_{i}\right)}{\sum_{i}^{M} w\left(\boldsymbol{R}_{i}\right)}
$$

where $E_{L}$ is a local energy, evaluated as

$$
E_{L}=\widehat{H} \Psi_{T}(\boldsymbol{R}) / \Psi_{T}(\boldsymbol{R})
$$

and the $w$ are weights at point $\boldsymbol{R}$. The $\boldsymbol{R}_{\boldsymbol{i}}$ are the configurations and they are distributed according to the mixed density, which in turn is calculated as a combination of the trial wavefunction and the exact one (estimated in DMC).

The trial wavefunctions, modified by the inclusion of a two-body Slater-Jastrow factor 38, were $\square$-optimized using the VMC method. DMC calculations were then performed, applying the Green's function technique 27 , using a 0.01 timestep and the Tmoves Casula's correction 39, aimed to go beyond the locality approximation in standard DMC and restoring the variational property in the computed energy. In the case of multideterminantal SOCI/CASSCF $(10,10)$ wavefunctions, configurations were included with different cutoff limits for the weights (from 0.1 to 0.001 ). In this case, the CI coefficients and the Jastrow parameters were optimized together. The QMCPACK program 40 was used to perform both the VMC and DMC calculations.

DMC works by producing an ensemble of replicates of the system, called walkers. The process consists in moving the electrons of each walker in imaginary time through Langevin diffusion (the propagator approach). The resulting configuration is subject to branching (a death-birth process). In these conditions, a DMC iterative process, if left to run for an infinite time, would lead to infinite number of walkers or none at all. For that reason, an artificial procedure is included, called population control 
bias, designed to yield a number of walkers that oscillates around a mean value 41 , or alternatively by fixing the number of walkers 42 , using weight-branching. In this work, population bias was tested using 9600, 19200, 38400 and 76800 configurations.

An alternative method is called reptation quantum Monte-Carlo (RMC) 43. In this case, the Metropolis algorithm is used to decide the branching (a radomly produced configuration is accepted or rejectd by the use of a threshold). RMC gives pure estimates of properties which operators do not conmute with the Hamiltonian. RMC is free from population bias and it is known from the literature to afford better results for observables than DMC in certain cases.

When analyzing the RMC results one has to keep in mind the meaning of the parameters that affect the quality of the RMC projection. On one side we have the imaginary projection time $\square$ for the sampling path (the size of the reptile) on the other the Trotter time step $\square . \square$ needs to be large enough so that the projections of the trial function $\Psi_{-} \mathrm{T}$ give a good aproximation to the true wavefunction, both for mixed and pure observables. The approximate equations to be fulfilled is

$$
e^{-\beta \widehat{H}}\left|\Psi_{T}\right\rangle \approx\left|\Phi_{0}\right\rangle
$$

The reptile is discretized into the number of bits $\mathrm{N}=\square \square \square$ called "beads" (an odd number is selected so that there is a central bead). This process gives an error in the observables that is directly proportional to $\square$, reason for which this parameter should be reasonably small. At the same time, the larger the $\square$ is, the more difficult is the calculation since larger reptiles have to be propagated.

In this work we used the RMC method with a 45 hartree- 1 reptile length, with the specific aim to get a better estimation of the dipole moment. Both RMC and DMC calculations of the dipole moment were performed with $\square=0.01$ hartree -1 , which was 
converged within error bars, and for our RMC calculations, we chose $\mathrm{N}=4501$ (which corresponds to the 45 hartree-1 long projection length). These calculations were performed using the QWalk code 44. Due to some limitations of this program, the QZ basis set used in the DMC calculations could not be employed and a TZ basis set was used instead.

\section{RESULTS AND DISCUSSION}

\section{Results obtained using MO and DFT methods}

PECs obtained at the HF, CCSD(T), CASSCF $(10,10)$ and MRCI/CASSCF $(10,10)$ levels, with the Karlsruhe basis sets, are shown in Figure 1. Only valence electrons were included in the $\operatorname{CCSD}(\mathrm{T})$ calculations, i.e. core correlation energy was not included. The second panel in the figure shows an enlargement of the region of the minima. All results are expressed in $\mathrm{kcal} / \mathrm{mol}$ with respect to the minimum of each curve, which is independently set to be $0.0 \mathrm{kcal} / \mathrm{mol}$.

(insert Figure 1 here)

As would be expected, the Hartree-Fock calculation leads to the erroneous dissociation limit. Post Hartree-Fock methods lead instead to the same correct dissociation limit and the PECs are so close among them as to be nearly indistinguishable. DFT methods exhibit an intermediate behavior between HF and $\operatorname{CCSD}(\mathrm{T})$. It does not lead to the correct dissociation limit, but the equilibrium distance is nearer to the post-Hartree-Fock results than to the HF one. Introduction of dispersion using the empirical GD3 correction does not improve the dissociation. On the contrary, it shows a spurious second minimum. This result, not reported earlier to the extent of 
our knowledge, implies that great care must be exercised in using the GD3 empirical correction in geometry optimizations, PECs or calculation of potential energy surfaces.

Equilibrium distances, dissociation energies, dipole moments and frequencies of the NaK molecule obtained with these methods are shown in Table I. The effect of the basis set on the results was tested only for the PBE0 and CCSD(T,Full) methods, taking values from the literature and those calculated in this work. In this less time consuming calculation we included all the electrons in the $\operatorname{CCSD}(\mathrm{T}$, Full) calculations.

\section{(insert Table 1 here)}

The best results of the equilibrium distance were obtained by Fedorov et al ${ }^{19}$ who also obtained good values for the dissociation energy, dipole moment and harmonic frequency. Surprisingly, the results of Zemke et al $^{15}$ are also quite good for the equilibrium distance, but much worse for the dipole moment. We tried to reproduce these results, using the same methodology, but obtained a larger equilibrium distance

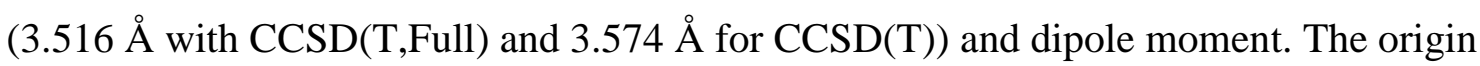
of this difference remains unknown. However, it is easy to see that there is a large variation of the results depending on the amount of dynamic correlation included and the size and type of the basis set.

The non-dynamic correlation seems not to be important, as could be expected. The inclusion of the core relativistic ECP do favor a better estimation of the dipole moment, while all electron calculations give a larger value. Our best calculation, $\operatorname{CCSD}(\mathrm{T}) /$ aug-cc-pc4, reproduces very well the equilibrium distance (-0.4\% error) and the harmonic vibrational frequency $(1.3 \%$ error) but exhibits a $13 \%$ error in the dipole moment. 
It is important to realize the effect that the inclusion of core electrons have in CCSD(T) calculations. Considering only the 6-311+G(3df) and Def2-QZVPPD basis sets, one can observe important variations between the CCSD(T,Full) and CCSD(T) calculations. In both cases, the too large bond distances obtained with $\operatorname{CCSD}(\mathrm{T}), 3.574$ and 3.665 A respectively, decrease to the much better 3.516 and $3.472 \AA$ when all electrons are correlated in the $\operatorname{CCSD}(\mathrm{T})$ calculations.

The increase of the basis set from Def2-TZPV to Def2-QZPVDD produces a shorter equilibrium distance and, more important, a decrease of the dipole moment of 0.6 D, nearer to the experimental result. The balance between polarization and diffuse functions seems to be extremely important to obtain this result, and the progression of the results in the series of correlation consistent basis sets Aug-pc-0, $(9 \mathrm{~s} 8 \mathrm{p}) /[6 \mathrm{~s} 4 \mathrm{p}]$, to Aug-pc-4, $(25 \mathrm{~s} 17 \mathrm{p} 9 \mathrm{~d} 3 \mathrm{f} 2 \mathrm{~g}) /[9 \mathrm{~s} 7 \mathrm{p} 6 \mathrm{~d} 3 \mathrm{f} 2 \mathrm{~g}]$, supports this assertion. Up to aug-pc-2, the equilibrium distance decreases, approaching the experimental value, and so does the dipole moment. However, the equilibrium distance obtained with the aug-pc-3 and augpc-4 basis sets is too short and the dipole moment bounces back, becoming again larger than 3 D. In our opinion, the good results reported for the dipole moment at the $\operatorname{CCSD}(\mathrm{T})$ and CCSDT levels is mostly due to error compensation within the basis sets and pseudopontials used. No convincing evidence exists as yet that the experimental dipole moment can be well predicted using a complete basis set extrapolation scheme.

\section{Results obtained using QMC methods}

The potential energy curves obtained using DMC with trial functions built at different theoretical levels and optimized using VMC are shown in Figure 2. Ross experimental curve $^{13}$ is included by way of comparison. 
Several curves are presented in this figure. The basic calculation is the 2-body Jastrow corrected Hartree-Fock trial function, which we called simply HF. To that, one can add the backflow correction ${ }^{27}$ and obtain the HF/BF curve. CISD, CASSCF and SOCI/CASSCF are also shown in the figure. As expected, introducing dynamical correlation energy corrects the form of the $\mathrm{QMC}(\mathrm{HF})$ curve. The $\mathrm{QMC}(\mathrm{CISD})$ and the QMC(SOCI/CASSCF) curves approximate the experimental dissociation from below and above respectively. Correction for the non-dynamical correlation energy through the multireference second order CI calculation does not modify the shape of the curve but shifts it upwards a bit. Comparing the experimental curve with those obtained by QMC, one sees a very reasonable agreement that does not depend greatly on the trial wavefunction used. Nonetheless, the dissociation energy does depend on the method, as can be seen in Table II.

(Insert Table II here)

The dissociation energy obtained with DFT trial wavefunctions is about $400 \mathrm{~cm}^{-}$ ${ }^{1}$ smaller than the experimental one. The HF trial wavefunction, gives a value which is about $250 \mathrm{~cm}^{-1}$ smaller than experiment. Including non-dynamical effects has a lower impact, and inclusion of dynamical correlation through the SOCI method on top of the CASSCF cancels all the effect. The origin of the remaining error, after including dynamical correlation, is not clear. However, one problem may be associated to the use of the pseudopotential. As the conventional $\operatorname{CCSD}(\mathrm{T})$ calculations show, the influence of the correlation energy of the core is important. Perhaps the remaining error is then due to the ECP and/or the use of a not so large basis sets. These are possible directions for improvement of the calculations. 
Expectation values of quantities which commute with the Hamiltonian are unbiased in DMC. However, expectation values of non-commuting operators, are estimated by mixed estimators and therefore they are not exact. Thus DMC has problems to predict dipole moments. ${ }^{45}$ One way to correct this drawback is to use RMC ${ }^{46}$, which we employed in this work with several trial wavefunctions. Due to the difficulty of the calculations, only the HF and DFT trial functions were used. The result obtained at the $\mathrm{RMC}(\mathrm{HF})$ level is not very encouraging, 3.0 D vs an experimental value of $2.72 \pm 0.05 \mathrm{D}$. However, the statistical error is quite large, $0.5 \mathrm{D}$, technically implying that the experimental value is within the error bounds. The values obtained at the $\mathrm{RMC}(\mathrm{B} 3 \mathrm{LYP})$ and $\mathrm{RMC}(\mathrm{PBE})$ level, $2.7 \pm 0.1 \mathrm{D}$ and $2.5 \pm 0.3 \mathrm{D}$ respectively, agree reasonably well with the experimental value.

Two interesting comparisons can be performed to assess the precision and accuracy of these calculations. On the one side, one can compare DMC and RMC values with the different trial functions (see later on the technical details on the way the RMC values of the dipole moment were calculated). The results show that the RMC and DMC results are close to the experimental value (see Table III). This, up to our knowledge, is one of the very few examples in the literature of a comparison of DMC and RMC values for dipole moments. In this respect, it must be mentioned the work of Wagner and Mitas ${ }^{47}$ where they calculated dipole moments of transition metal monoxides and pointed out that the dipole moment remains an extraordinary sensitive quantity that is a stringent test for theory. In a similar work using DMC Guo et al. ${ }^{45}$ studied the dipole moment of both $\mathrm{LiSr}$ and $\mathrm{KRb}$ and found reasonable agreement in the second case, but not the first (about half the value predicted by conventional methods). RMC seems to be helpful in solving this problem. 
We followed the work of Rothstein ${ }^{45}$ who performed RMC calculations using the B3LYP wavefunction and analyzing the convergence of energy and dipole moment using different time steps and reptile size. We used six values for the reptile size (24, $32,40,48,64,80)$ and, for each value, we varied the time step and number of beads so that the former was kept constant. We chose the values o $0.0025,0.0050,0.0075$, $0.0100,0.0150$ and 0.0200 for the time step. The energy of the system converged for all sizes of the reptile. However, the dipole moment converged only for the largest sizes. Meaningful data were obtained for 80 Hartree $^{-1}$ and for this time step five independent calculations were run. The average value of these runs yielded a zero time-step extrapolation of 2.7(2) D. This value is shown in Table III together with our own values obtained with other methodologies and the experimental one

\section{(Insert Table III here)}

While there is a big difference between the DMC results using several trial wavefunctions, the RMC results have a smaller dispersion. Both the RMC/HF [2.5-3.5] and RMC/B3LYP [2.5-2.9] calculations afford results that encompass the experimental value. Both values are even better that the $\operatorname{CSSD}(\mathrm{T})$ result with the most extended basis set performed in this paper. We already mentioned that better results shown in the literature using $\operatorname{CCSD}(\mathrm{T})$ exhibit error compensation between the method and the pseudopotential included in the basis set. Even when using a very simple trial wavefunction, as Hartree-Fock, both DMC and RMC results are better than simple conventional HF calculations.

The second comparison that can be done refers to the known problem of ergodicity in RMC. Due to the algorithms used, it is possible for the reptiles to bounce 
back and forth without changing the electron coordinates of the central bead (as described in the QMCPack code manual). Thus, strong autocorrelation effects may be occurring, and this effect should be checked. We actually found that these effects were present in our calculations. To reduce them as much as possible we increase the sampling period used in the reblocking method. Thus, the procedure consisted in calculating the average from more spaced configurations when the time step between configurations decreased. This procedure provided the necessary decorrelation of the averages.

\section{Conclusions}

Quantum Monte Carlo calculations of the equilibrium distance, dissociation energy and dipole moment of $\mathrm{NaK}$ have been performed using both DMC and RMC methods. CCSD(T) calculations, as well as CASSCF and MRCI, using several basis sets were performed to use as a comparison. It was found that the dipole moment of $\mathrm{NaK}$ is very sensitive to the size and composition of the basis set. No systematic trend was found in the results obtained, even if progressively larger and more complete basis sets were used. It was nonetheless shown that dynamical correlation energy is more important than non-dynamical correlation energy in the calculation of both the dissociation energy and dipole moment. Inclusion of core electrons in the calculation of the correlation energy does have a very noticeable effect in the equilibrium distance. Good results obtained in other calculations seem to be an effect of error compensation between the method, the basis set and the use of pseudopotentials.

The QMC results obtained showed that the dissociation energy was overestimated when a HF trial function was employed. Use of a CISD wavefunction built on top of the HF wavefunction lead instead to an underestimation of the result. The use of a SOCI/CASSCF trial wavefunction did not afford any improvement in the 
results. The dissociation energies obtained with conventional MO methods are better than those obtained with QMC. Whether this disagreement can be corrected by use of larger basis sets, better trial wavefunctions or longer times is a matter of speculation. Moreover, the use of the Burkatski Filippi Dolg ECP means that only one electron is preserved in the description of Na. Thus, one can not rule out that the error may be in the use of the ECP.

On the contrary, the dipole moment obtained using the RMC method with as simple a trial wavefunction as $\mathrm{HF}$ is as good as the value obtained with the best $\operatorname{CCSD}(\mathrm{T})$ calculation performed in this paper. These results, however, can not be systematically improved, while the QMC can be better if longer simulations or better trial wavefunctions are used. Actually, using DFT trial wavefunctions, like B3LYP, the resulting dipole moment is well in agreement with the experimental value.

\section{Acknowledgments}

The funding agencies Pedeciba, CSIC and ANII are gratefully acknowledged for partial funding of this research.

\section{References}

1. Yelin, S. F.; Kirby, K.; Côté, R. Schemes for robust quantum computation with polar molecules. Phys. Rev. A 2006, 74, 050301 (R).

2. Kuznetsova, E.; Côté, R.; Kirby, K.; Yelin, S. F. Analysis of experimental feasibility of polar-molecule-based phase gates. Phys. Rev. A 2008, 78, 012313.

3. Vitanov, N. V.; Fleischhauer, M.; Shore, B. W.; Bergmann, K. Coherent manipulation of atoms and molecules by sequential laser pulses. Adv. At. Molec. Opt. Phys. 2001, 46, 55-190.

4. Pellegrini, P.; Gacesa, M.; Côté, R. Giant formation rates of ultracold molecules via Feshbach-optimized photoassociation. Phys. Rev. Lett. 2008, 101, 053201. 
5. Deiglmayr, J.; Grochola, A.; Repp, M.; Mörtlbauer, K.; Glück, C.; Lange, J.; Dulieu, O.; Wester, R.; Weidemüller, M. Formation of Ultracold Polar Molecules in the Rovibrational Ground State. Phys. Rev. Lett. 2008, 101, 133004.

6. Klincare, I.; Nikolayeva, O.; Tamanis, M.; Ferber, R.; Pazyuk, E. A.; Stolyarov, A. $\mathrm{V}$. Modeling of the $\mathrm{X} 1 \Sigma+$, a $3 \Sigma+\rightarrow \mathrm{E}(4) 1 \Sigma+\rightarrow \mathrm{X} 1 \Sigma+(\mathrm{v}=0, \mathrm{~J}=0)$ optical cycle for ultracold KCs molecule production. Phys. Rev. A 2012, 85, 062520.

7. Ospelkaus, S.; Ni, K.-K.; Wang, D.; de Miranda, M. H. G.; Neyenhuis, B.; Quéméner, G.; Julienne, P. S.; Bohn, J. L.; Jin, D. S.; J. Ye, J. QuantumState Controlled Chemical Reactions of Ultracold Potassium-Rubidium Molecules. Science 2010, 327, 853-857.

8. Rossa, A. J.; Effantina, C.; d'Incana, J.; Barrow, R. F. Long-range potentials for the X $1 \Sigma+$ and a $3 \Sigma+$ states of the NaK molecule. Mol. Phys. 1985, 56, 903-912.

9. Wormsbecher, R. F.; Hessel, M. M.; Lovas, F. J. Microwave optical double

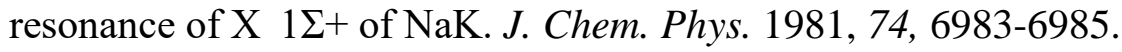

10. Yamada, C.; Hirota, E. Microwave Spectroscopy of NaK. J. Mol. Spectrosc. 1992, 153, 91-95.

11. Krou-Adohi, A.; Giraud-Cotton, S. The Ground State X 1S+ of NaK Revisited. J. Mol. Spectrosc. 1998, 190, 171-188.

12. Dagdigian, P. J.; Wharton, L. Molecular Beam Electric Deflection and Resonance Spectroscopy of the Heteronuclear Alkali Dimers: 39K7Li, Rb7Li, 39K23Na, Rb23Na, and 133Cs23Na. J. Chem. Phys. 1972, 57, 1487-1496.

13. Russier-Antoine, I.; Ross, A. J.; Aubert-Frécon, M.; Martin, F.; Crozet, P. An improved potential energy curve for the ground state of NaK. J. Phys. B: At. Mol. Opt. Phys. 2000, 33, 2753-2762.

14. Gerdesa, A.; Hobein, M.; Knöckel, H.; Tiemann, E. Ground state potentials of the NaK molecule. Eur. Phys. J. D 2008, 49, 67-73.

15. Zemke, W. T.; Byrd, J. N.; Michels, H.; Montgomery, J. . J. A.; Stwalley, W. C. Long range intermolecular interactions between the alkali diatomics $\mathrm{Na}(2)$, K (2), and NaK. J. Chem. Phys. 2010, 132, 244305.

16. Byrd, J. N.; Montgomery, J. . J. A.; Côté, R. Structure and thermochemistry of K2Rb, KRb2, and K2Rb2. Phys. Rev. A 2010, 82, 010502(R). 
17. Jason N. Byrd, J. N.; Côté, R.; Montgomery Jr., J. A. Long-range interactions between like homonuclear alkali metal diatoms. J. Chem. Phys. 2011, 135, 244307.

18. Aymar, M.; Dulieu, O. Calculation of accurate permanent dipole moments of the lowest $1,3 \Sigma+$ states of heteronuclear alkali dimers using extended basis sets. J. Chem. Phys. 2005, 122, 204302.

19. Fedorov, D. A.; Derevianko, A.; Varganov, S. A. Accurate potential energy, dipole moment curves, and lifetimes of vibrational states of heteronuclear alkali dimers. J. Chem. Phys. 2014, 140, 184315.

20. Weigend, F.; Furche, F.; Ahlrichs, R. Gaussian basis sets of quadruple zeta valence quality for atoms H-Kr. J. Chem. Phys. 2003, 119, 12753.

21. Adamo, C.; Barone, C. Toward reliable density functional methods without adjustable parameters: The PBE0 model. J. Chem. Phys. 1999, 110, 61586169.

22. Grimme, S.; Antony, J.; Ehrlich, S.; Krieg, H. A consistent and accurate ab initio parameterization of density functional dispersion correction (DFT-D) for the 94 elements H-Pu. J. Chem. Phys. 2010, 132, 154104.

23. Frisch, M. J.; Trucks, G. W.; Schlegel, H. B.; et al. Gaussian 09, Revision D.01. Gaussian, Inc., Wallingford CT 2009.

24. Werner, H.-J.; Knowles, P. J.; Lindh, R.; Manby, F. R. et al. Molpro, version 2009.1, a package of ab initio programs. 2009.

25. Jensen, F. Polarization consistent basis sets. VI. The elements $\mathrm{He}, \mathrm{Li}, \mathrm{Be}, \mathrm{B}, \mathrm{Ne}, \mathrm{Na}$, Mg, Al, Ar. J. Phys. Chem. A 2007, 111, 11198-11204.

26. Jensen, F. Polarization Consistent Basis Sets. VII. The Elements K, Ca, Ga, Ge, As, Se, Br and Kr. J. Chem. Phys. 2012, 136, 094110.

27. Foulkes, W. M. C.; Mitas, L.; Needs, R. J.; Rajagopal, G. Quantum Monte Carlo simulations of solids. Rev. Mod. Phys. 2001, 73, 33-83.

28. Hammond, B. L.; Lester Jr., W. A.; Reynolds, P. J. Monte Carlo Methods and Ab Initio Quantum Chemistry; World Scientific: Singapore, 1994.

29. Anderson, J. B., Rothstein, S. M., Eds. Advances in Quantum Monte Carlo; American Chemical Society: Washington, 2007. 
30. Anderson, J. B. Quantum Monte Carlo: Origins, Development, Applications;

Oxford: New York, 2007.

31. Huang, P.; Carter, E. A. Advances in Correlated Electronic Structure Methods for Solids, Surfaces, and Nanostructures. Annu. Rev. Phys. Chem. 2008, 59, 261290.

32. Lester, J. . W. A.; Mitas, L.; Hammond, B. Quantum Monte Carlo for atoms, molecules and solids. Chem. Phys. Lett. 2009, 478, 1-10.

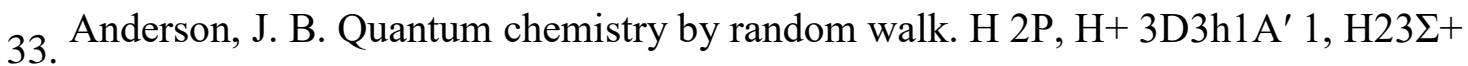

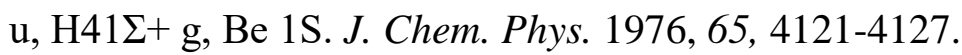

34. Reynolds, P. J.; Ceperley, D. M.; Alder, B. J.; Lester Jr, W. A. Fixed-node quantum Monte Carlo for molecules. J. Chem. Phys. 1982, 77, 5593-5603.

35. Morales, M. A.; McMinis, J.; Clark, B. K.; Kim, J.; Scuseria, G. E. Multideterminant Wave Functions in Quantum Monte Carlo. J. Chem. Theor. Comput. 2012, 8, 2181-2188.

36. (a) Burkatzki, M.; Filippi, C.; Dolg, M. Energy-consistent pseudopotentials for quantum Monte Carlo calculations. J. Chem. Phys. 2007, 126, 234105. (b) Burkatzki, M.; Filippi, C.; Dolg, M. Energy-consistent small-core pseudopotentials for 3d-transition metals adapted to quantum Monte Carlo calculations. J. Chem. Phys. 2008, 129, 164115 (2008).

37. Kim, J.; Esler, K. P.; McMinis, J.; Morales, M. A.; Clark, B. K.; Shulenburger, L.; Ceperley, D. M. Hybrid algorithms in quantum Monte Carlo. J. Phys.: Conference Series 2012, 402, 012008.

38. Toulouse, J.; Umrigar, C. J. Full optimization of Jastrow-Slater wave functions with application to the first-row atoms and homonuclear diatomic molecules. $J$. Chem. Phys. 2008, 128, 174101.

39. Casula, M. Beyond the locality approximation in the standard diffusion Monte Carlo method. Phys. Rev. B 2006, 74, 161102(R).

40. Jeongnim Kim, K. E. J.; Clark, B.; Gergely, J.; Chiesa, S.; Delaney, K.; Vincent, J.; Ceperley, D. QMCPACK Simulation Package, version QMCPACK_rev6854_20160404.http://qmcpack.org/2016.

41. Vrbik, J.; Rothstein, S. M. Quadratic accuracy diffusion Monte Carlo. J. Comput. Phys. 1986, 63, 130-139. 
42. Bosá, I.; Rothstein, S. M. Unbiased expectation values from diffusion quantum Monte Carlo simulations with a fixed number of walkers. J. Chem. Phys. 2004, 121, 4486-4493.

43. Baroni, S.; Moroni, S. Reptation quantum Monte Carlo: a method for unbiased ground-state averages and imaginary-time correlations. Phys. Rev. Lett. 1999, 82, 4745-4748.

44. Wagner, L. A.; Bajdich, M.; Mitas, L. QWalk: A quantum Monte Carlo program for electronic structure. J. Comput. Phys. 2009, 228, 3390-3404.

45. Guo, S.; Bajdich, M.; Mitas, L.; Reynolds, P. J. Study of dipole moments of LiSr and KRb molecules by quantum Monte Carlo methods. Mol. Phys. 2013, $111,1744-1752$.

46. Per, M.; Sook, I. K.; Russo, S. P. Efficient calculation of unbiased expectation values in diffusion quantum Monte Carlo. Phys. Rev. B 2012, 86, 201107.

47. Wagner, L. K.; Mitas, L. Energetics and dipole moment of transition metal monoxides by quantum Monte Carlo. J. Chem. Phys. 2007, 126, 034105.

\section{Heading 1: use this style for level one headings}

Display quotations of over 40 words, or as needed.

- For bulleted lists

(1) For numbered lists 
TABLE I. Equilibrium bond distances (in $\AA$ ), dissociation energies (in $\mathrm{cm}^{-1}$ ), dipole moments (in D) and harmonic vibrational frequencies (in $\mathrm{cm}^{-1}$ ) calculated for the $\mathrm{NaK}$ molecule using molecular orbital and DFT methods.

\begin{tabular}{|c|c|c|c|c|c|c|}
\hline Method & Basis set & $\mathrm{R}_{\mathrm{e}}$ & $\Delta \mathrm{E}$ & $\mu$ & $\omega_{\mathrm{e}}$ & Reference \\
\hline $\mathrm{HF}$ & Def2-QZVPPD & 3.263 & 12893 & 6.71 & 156.6 & This work \\
\hline \multirow[t]{2}{*}{ PBE0 } & Def2-QZVPPD & 3.549 & 10768 & 3.02 & 123.0 & This work \\
\hline & pc-4 & 3.546 & & 3.01 & 123.3 & This work \\
\hline PBE0-GD3 & Def2-QZVPPD & 3.571 & 10767 & 3.04 & 118.1 & This work \\
\hline CASSCF & Def2-QZVPPD & 3.265 & 5087 & 7.39 & 110.0 & This work \\
\hline MRCI & Def2-QZVPPD & 3.049 & 5171 & 3.93 & 118.6 & This work \\
\hline \multirow[t]{10}{*}{ CCSD(T,Full) } & Def2-QZVPPD & 3.472 & 5262 & 3.06 & 126.7 & This work \\
\hline & Def2-QZVP & 3.461 & & 3.70 & 127.4 & This work \\
\hline & Def2-TZVP & 3.529 & & 3.65 & 121.1 & This work \\
\hline & $6-311+G(3 d f)$ & 3.516 & & 3.15 & 123.2 & This work \\
\hline & pc-4 & 3.479 & & 3.10 & 122.7 & This work \\
\hline & Aug-pc-0 & 3.652 & & 4.23 & 114.3 & This work \\
\hline & Aug-pc-1 & 3.608 & & 3.05 & 119.3 & This work \\
\hline & Aug-pc-2 & 3.516 & & 2.98 & 120.1 & This work \\
\hline & Aug-pc-3 & 3.491 & & 3.12 & 126.2 & This work \\
\hline & Aug-pc-4 & 3.484 & 5270 & 3.11 & 125.7 & This work \\
\hline \multirow[t]{2}{*}{$\operatorname{CCSD}(\mathrm{T})$} & $6-311+G(3 d f)$ & 3.488 & & 2.94 & & 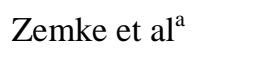 \\
\hline & Pseudopotential+extended & 3.440 & & 2.76 & & Aymar \& Dulieu ${ }^{b}$ \\
\hline CCSDT & Pseudopotential+QZ & 3.504 & 5364 & 2.68 & 122.4 & Fedorov et al $^{\mathrm{d}}$ \\
\hline \multirow[t]{3}{*}{ Experimental } & & 3.499 & 5273.62 & & & 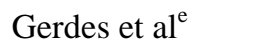 \\
\hline & & & & 2.76 & & Dagdigian et $\mathrm{al}^{\mathrm{f}}$ \\
\hline & & & & & 124.13 & Wormsbecher ${ }^{\mathrm{g}}$ \\
\hline
\end{tabular}

${ }^{a}$ Reference 15. ${ }^{\mathrm{b}} \mathrm{cc}-\mathrm{pCVQZ}$ for Na and small-core relativistic effective core potentials (ECP) of Stuttgart/Cologne group for K. ${ }^{\mathrm{c}}$ Reference 18. ${ }^{\mathrm{d}}$ Reference 19. ${ }^{\mathrm{e}}$ Reference 14. ${ }^{\mathrm{f}}$ Reference 12. ${ }^{\mathrm{g}}$ Reference 9 
TABLE II. Equilibrium distances (in $\AA$ ) and dissociation energies (in $\mathrm{cm}^{-1}$ ) calculated using different methods, trial functions and correction factors. Statistical error reported also in $\mathrm{cm}^{-1}$

\begin{tabular}{|c|c|c|c|c|c|c|}
\hline Method & Trial function & Basis Set & Improvement & $\mathrm{r}_{\mathrm{e}}$ & $\Delta \mathrm{E}$ & Statistical Error \\
\hline \multirow[t]{8}{*}{$\overline{\mathrm{DMC}}$} & BHHLYP & & Jastrow & & 4850 & 44 \\
\hline & PBE & & Jastrow & & 4828 & 66 \\
\hline & B3LYP & & Jastrow & & 4872 & 66 \\
\hline & $\mathrm{HF}$ & VQZ/ANO & Jastrow $^{\text {a }}$ & 3.535 & 5026 & 219 \\
\hline & & VQZ/ANO & Jastrow+Backflow ${ }^{\mathrm{a}}$ & 3.530 & 5662 & 66 \\
\hline & CISD & VQZ/ANO & Jastrow & 3.525 & 5048 & 219 \\
\hline & CASSCF & VQZ/ANO & Jastrow & 3.530 & 5531 & 44 \\
\hline & SOCI/CASSCF & VQZ/ANO & Jastrow & 3.530 & 5717 & 44 \\
\hline RMC & $\mathrm{HF}$ & $\mathrm{TZV}$ & Jastrow & & 5706 & 220 \\
\hline $\operatorname{CCSD}(T)^{\mathrm{b}}$ & & Def2-QZVPPD & & 3.472 & 5262 & \\
\hline Experimental $^{\mathrm{a}}$ & & & & 3.499 & 5273.62 & \\
\hline
\end{tabular}

${ }^{a}$ Due to the incorrect behavior at large distances, in this case the dissociation energy was evaluated taking as limit energy that at $r_{e}=8.0 \AA$

${ }^{\mathrm{b}}$ Reference 14.

${ }^{c}$ Result obtained by conventional MO calculations with the CCSD(T) method and the Def2QZVPPD basis set, as reported in Table I. 
TABLE III. Dipole moment (in D) calculated using different methods, basis sets and trial functions, compared to the experimental value.

\begin{tabular}{|c|c|c|}
\hline Method & Trial function (basis set) ${ }^{\mathrm{a}}$ & Dipole moment (D) \\
\hline \multirow[t]{3}{*}{$\overline{\mathrm{DMC}^{\mathrm{b}}}$} & $\mathrm{HF}(\mathrm{SJ})$ & $1.6 \pm 0.1$ \\
\hline & $\mathrm{PBE}(\mathrm{SJ})$ & $2.8 \pm 0.1$ \\
\hline & B3LYP(SJ) & $3.1 \pm 0.1$ \\
\hline \multirow[t]{3}{*}{$\mathrm{RMC}$} & $\mathrm{HF}(\mathrm{SJ})$ & $3.0 \pm 0.5$ \\
\hline & $\mathrm{PBE}(\mathrm{SJ})$ & $2.1 \pm 0.3$ \\
\hline & $\mathrm{B} 3 \mathrm{LYP}(\mathrm{SJ})$ & $2.7 \pm 0.2$ \\
\hline $\mathrm{HF}$ & Def2-QZVPPD & 6.71 \\
\hline PBE0 & Def2-QZVPPD & 3.02 \\
\hline CASSCF & Def2-QZVPPD & 7.39 \\
\hline MRCI & Def2-QZVPPD & 3.93 \\
\hline $\operatorname{CCSD}(\mathrm{T})$ & Def2-QZVPPD & 3.06 \\
\hline & Aug-pc-4 & 3.11 \\
\hline Experimental $^{\mathrm{b}}$ & & $2.76 \pm 0.05$ \\
\hline
\end{tabular}

${ }^{\mathrm{a}}$ Trial function applies for MC calculations; basis set applies to conventional DFT or MO calculations

${ }^{\mathrm{b}}$ The linear correction in DMC (extrapolated estimator = 2 DMC $-\mathrm{VMC}$ ) was used to reduce the DMC bias due to the non locality of the dipole operator.

${ }^{\mathrm{c}}$ Reference $\mathbf{1 2 .}$ 


\section{Figures}

FIG. 1. PECs for NaK at different levels of calculation, using the Kalsruhe Def2-

QZVPPD basis set. (b) is a close-up of the PECS in (a).

FIG. 2. PECs calculated using DMC method with a VQZ/ANO basis set. The

experimental curve of Ross is included as a comparison. (b) is a close-up of (a) in the region of the minima. 
FIGURE 1
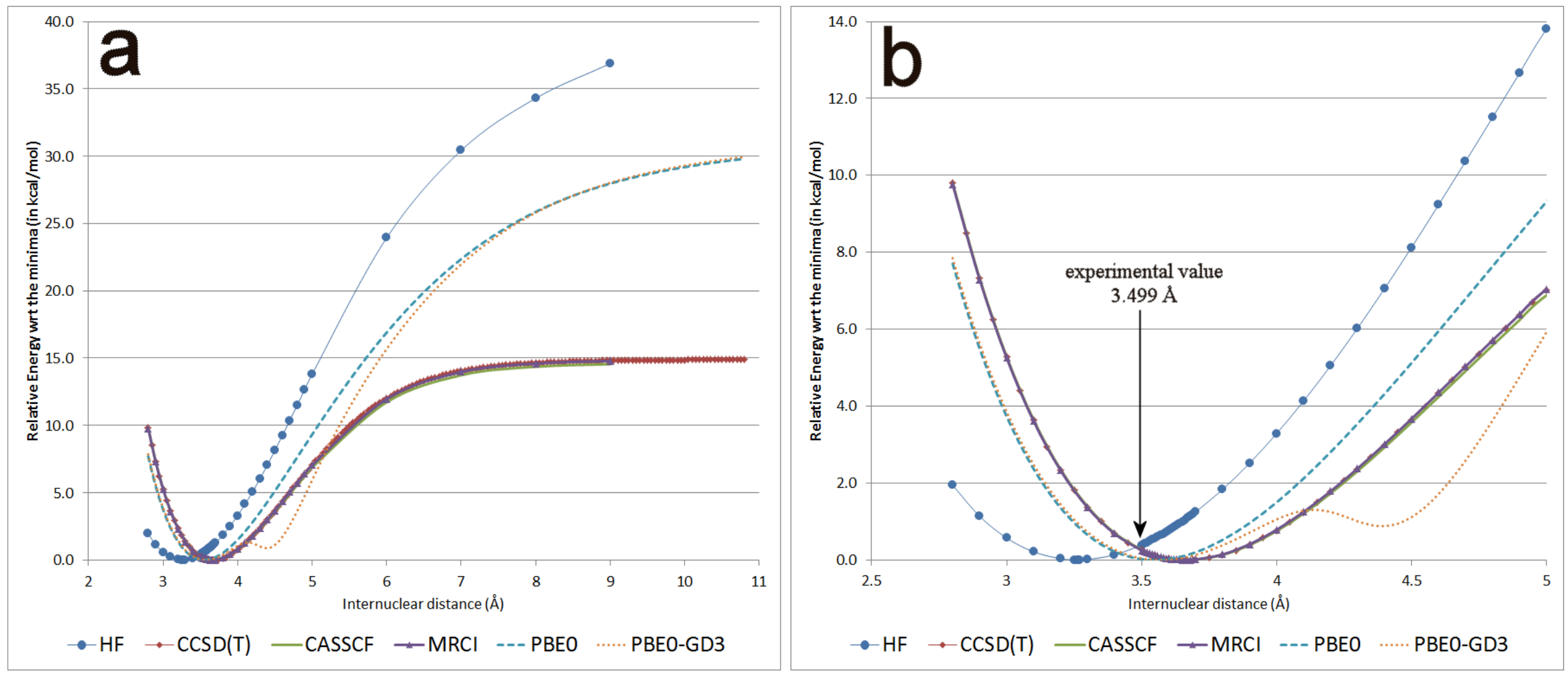
FIGURE 2
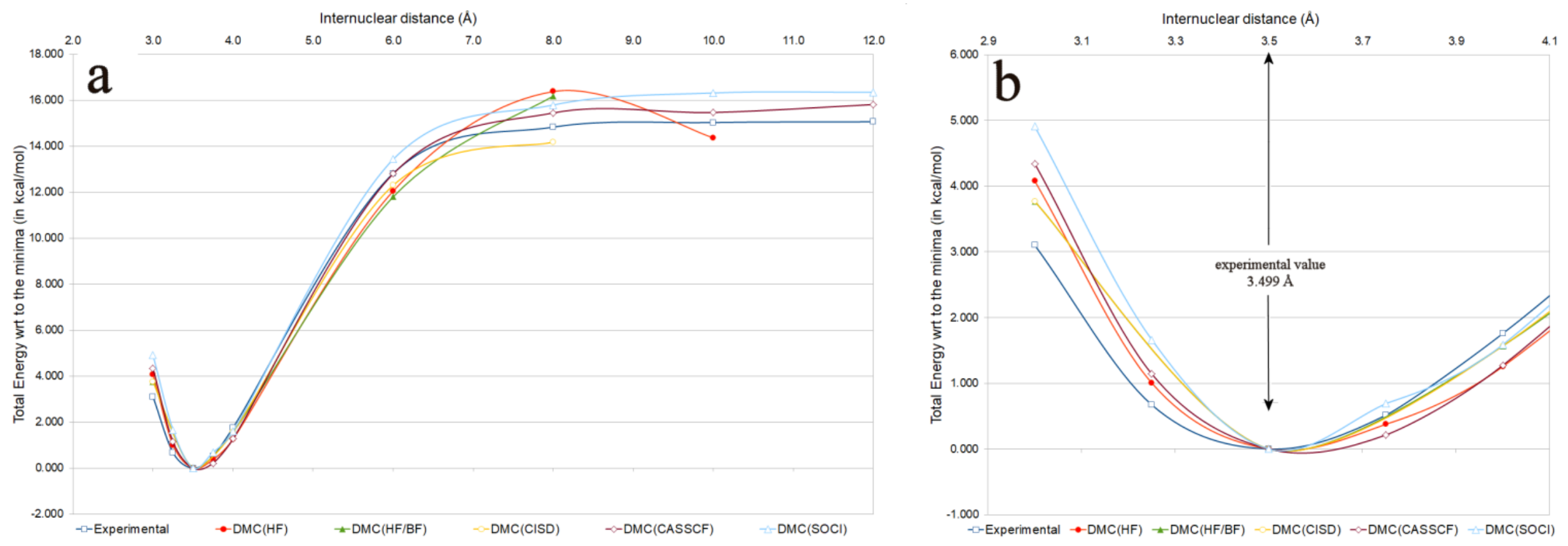